This work has been accepted to the IEEE for publication. Copyright has been transferred to IEEE. To cite this work, please use the following link.

doi.org/10.1109/TNSRE.2021.3131466 


\title{
A-GAS: a Probabilistic Approach for Generating Automated Gait Assessment Score for Cerebral Palsy Children
}

\author{
Rishabh Bajpai, Graduate Student Member, IEEE, and Deepak Joshi
}

\begin{abstract}
Gait disorders in children with cerebral palsy (CP) affect their mental, physical, economic, and social lives. Gait assessment is one of the essential steps of gait management. It has been widely used for clinical decision making and evaluation of different treatment outcomes. However, most of the present methods of gait assessment are subjective, less sensitive to small pathological changes, time-taking and need a great effort of an expert. This work proposes an automated, comprehensive gait assessment score (A-GAS) for gait disorders in CP. Kinematic data of $356 \mathrm{CP}$ and 41 typically developing subjects is used to validate the performance of A-GAS. For the computation of AGAS, instance abnormality index (AII) and abnormality index (AI) are calculated. AII quantifies gait abnormality of a gait cycle instance, while AI quantifies gait abnormality of a joint angle profile during walking. AII is calculated for all gait cycle instances by performing probabilistic and statistical analyses. Abnormality index (AI) is a weighted sum of AII, computed for each joint angle profile. A-GAS is a weighted sum of AI, calculated for a lower limb. Moreover, a graphical representation of the gait assessment report, including AII, AI, and A-GAS is generated for providing a better depiction of the assessment score. Furthermore, the work compares A-GAS with a present rating-based gait assessment scores to understand fundamental differences. Finally, A-GAS's performance is verified for a high-cost multi-camera set-up using nine joint angle profiles and a low-cost single camera set-up using three joint angle profiles. Results show no significant differences in performance of A-GAS for both the set-ups. Therefore, A-GAS for both the set-ups can be used interchangeably.
\end{abstract}

Index Terms-Human locomotion, gait assessment, cerebral palsy, automation

\section{INTRODUCTION}

A BNORMAL bone growth, bone deformation, loss or reduction of neural activation in muscles, loss of proprioception and muscle abnormalities may lead to abnormal walking patterns [1]. Cerebral Palsy (CP) is the most common pediatric neurodevelopmental disorder that affects motor skills, muscle tones, balance and movements [2]. Since CP is a non-progressive disorder, accurate and timely gait management may help in significant recovery of the movements. Assessment of abnormal walking patterns is considered one of the most crucial steps of gait management in CP. Accurate assessment of gait helps the doctor in selecting appropriate interventions [3]. For example, in case of minor gait deviations with spasticity, botulinum toxin may be provided for relaxing

R. Bajpai and D. Joshi are with Indian Institute of Technology Delhi,110016, India, and All India Institute of Medical Science Delhi, New Delhi,110029, India. E-mail: \{bmz208129@iitd.ac.in, rishabhbajpai24@gmail.com\},joshid@iitd.ac.in.

Manuscript received ; muscles and avoiding muscle contracture [4]. Then, a series of physical therapy sessions are conducted to strengthen the muscles. In between the sessions, a gait assessment is performed to track the changes in gait and formulate new management strategies. However, most of the present gait assessment tools are not very sensitive to record small pathological changes in the gait [5]. The following subsection overviews present gait assessment tools by explaining their assessment methodologies, clinical relevance, limitations and advantages over other methods.

\section{A. Literature Review}

Broadly, gait assessment tools can be classified into three categories: qualitative methods, quantitative methods, and clinical gait analysis. Qualitative methods of gait assessment include both observation-based methods and questionnaire-based methods [6]. The fundamental principle of observation-based methods is to detect and understand the abnormality in the gait, mostly by visualising the video of gait. Therefore, these methods demand a great understanding of the biomechanics of walking and experience in gait assessment. The observer generates a report, and calculates a gait assessment score based on the observations of gait's basic parameters such as stride, joint angles, posture and movements. Most of the popular observation-based classifications systems are based on the work of Sutherland et al. [7], Winter et al. [8] and Rodda et al. [9]. On the other hand, questionnaire-based methods need less understanding of walking and no experience in gait assessment. These methods consist of questions related to walking and other movements of the lower limbs. Gillette Functional Assessment Walking Scale is a popular 10-point scale parent-report questionnaire that can be used with no or little knowledge of gait [10]. Functional Mobility Scale [11], Gross Motor Function Classification System (GMFCS) [12], Pediatric Quality of Life inventory [13], Pediatric Evaluation of Disability Inventory [14], Gait Outcomes Assessment List [15] and Activities Scale for Kids [16] are some other widely used questionnaire-based assessments. No doubt, these methods are beneficial for preliminary gait assessment in the absence of an expert. However, they are less accurate, less comprehensive, and biased towards some common gait pathologies.

Most of the shortcomings of qualitative methods are addressed by quantitative methods. In these methods, gait data, mostly kinematics of joints and segments of a patient's lower limb, are 
compared with normal kinematics data for understanding areas of abnormality in the gait. The two most common quantitative approaches involve manual assessment score calculation and automatic computation based methods. In manual calculationbased methods, an expert (also known as rater) rates gaitrelated parameters by comparing them with their corresponding normal parameters values. For example, Edinburgh Visual Gait Score [17] was developed for assessing the gait of $\mathrm{CP}$ children in all three planes. It uses 17 gait parameters of ankle, knee, hip, pelvis and trunk, covering all three planes. It uses a three-point scale for each parameter, where ' 0 ' denotes normal, ' 1 ' denotes moderate deviation and ' 2 ' denotes marked deviation. After calculating ratings for all parameters, a gross rating is calculated by summing the individual ratings. As a result, the range of gross rating's value becomes $0-34$. One key limitation of this method is its subjectivity, due to which the ratings calculated for the same patient may vary with the raters [18]-[20]. Observational Gait Scale [21] is a manual calculation-based assessment score developed for assessing the gait of CP children in all three planes. It is based on twenty-six parameters (features) of gait, related to ankle/foot, knee, hip, pelvis joints. Physician's Rating Scale [22] and Salford Gait Tool [23] are two other popular scores to assess ankle, knee and hip joints. Physician's Rating Scale has many variants, namely, modified PRS [22], Abbreviated PRS [24], Visual Gait Analysis Scale [25] and observational gait scale [26]. All are based on a scoring system with a maximum score ranging from 1 to 26. Salford Gait Tool [23] is a five-point scale that assesses three joints at six gait events: initial contact, end of double support, midstance, start of double support, toe-off, and mid-swing. Manual calculation-based methods are beneficial in clinical settings. However, there are some major limitations of these methods discussed by many researchers in the last few decades [5], [18]-[20], [27].

On the other hand, automatic computational methods are objective because they generally use dimension reduction of the gait data to compute the gait assessment score and classify gait into normal and abnormal gait. Recently, Lei Wang et al. [28] proposed an inertial measurement unit (IMU) based gait normalcy index. The normalcy index was calculated from six kinematic parameters and six three spatial-temporal parameters extracted from two shank-mounted IMUs [28]. IMUbased methods can accurately extract only a smaller number of gait pathology relevant parameters. This limits the usability of IMU-based methods in clinical gait analysis. Similarly, Jing Gao et al. [29] proposed an IMU-based abnormal gait recognition algorithm. Their algorithm uses a long shortterm memory network and convolutional neural network for detecting tiptoe, hemiplegic, and cross-threshold gait abnormal gait. Their method is cost-efficient and can be used for the recognition of a set of abnormal gaits. However, every patient has a unique gait abnormality [1], and it is not feasible to train a neural network for all possible gait disorders. Trong-Nguyen Nguyen and Jean Meunier [30] proposed an approach that estimates a human walking gait abnormality index using an adversarial auto-encoder. They fit 3-D cloud data to cylinders and fed these cylinders to an adversarial auto-encoder to calculate some potential gait abnormality indices. In their other work [31], they tried to estimate skeleton-based gait abnormality index by sparse deep auto-encoder. Xiang Chen et al. [32] proposed a three IMUs based objective motion disability tool for CP patients. They compared gait acceleration data from three IMUs placed on the trunk and thighs of healthy and CP subjects to calculate a global score. The field of use of automatic computational methods for gait assessment is in its embryo stage. Present methods either provide a global abnormality index or perform classification into a small number of gait abnormalities.

Clinical gait analysis is considered the most comprehensive gait assessment procedure. It may include the processing of various gait-related signals like three-dimensional motion data (force, kinematic, kinetic of joints), electromyography (EMG), physical examination, CT scan and magnetic resonance imaging (MRI) [33]. This approach is time-consuming, subjective, detailed and needs a team of experts from various domains. It is recommended mainly in severe cases of gait abnormality [33].

\section{B. Motivation and Contributions}

As discussed above, a large literature can be found on the development, verification and implementation of gait assessment tools in clinical environment. No doubt, these methods can explain gait abnormalities in most cases [6]. However, there are four major limitations of these methods. First, most of these methods need significant efforts of an experienced evaluator. Second, they are subjective and prone to misclassification. Third, most of them use features of gait for assessment, making them less sensitive to detect minor changes in gait and capture uncommon gait abnormalities. Fourth, they are less user-friendly as they provide either expanded raw data that is difficult to interpret or provide some global indices, making the process of localisation of abnormality more difficult. Many studies highlighted the shortcomings of the present gait assessment method [18], [34]-[36]. Several studies reported large variability of assessment scores when a single subject had been analysed in a number of different laboratories [37], [38]. However, these methods are being used in clinical settings due to their popularity, intuitiveness and unavailability of a comprehensive automated gait assessment system. After understanding the merits, de-merits of the present methods of gait assessment and the needs of the clinicians, we concluded the following expectation from a gait assessment tool. The gait assessment should:

- be objective, comprehensive, easily implementable and user-friendly.

- use easy mathematics and white-box signal processing where inner components and logics are available for inspection. Such gait assessment tools would provide a better understanding of background processing to doctors/clinicians.

- use a continuous-valued scale for better sensitivity to minor changes in gait patterns.

- hold completeness property. By considering all lower limb joints namely, pelvic, hip, knee and ankle, and multiple gait cycle instances the completeness of gait assessment can be assured. 
- give freedom to the user to manipulate parameters used for the gait assessment.

- be fast. By using present methods, each session of gait assessment and interpretation of the results by experts takes three to six hours [39].

This work attempts to address above mentioned needs with the following significant contributions:

1) Automated-Gait Assessment Score (A-GAS) is the first objective, comprehensive gait assessment methodology developed for CP children (as per the authors' knowledge).

2) The work presents A-GAS report having three components corresponding to different levels of gait analysis. The first component depicts the global abnormality index of a limb, the second component depicts the abnormality index of each joint, and the third component depicts the abnormality of each joint at multiple gait cycle instances.

3) For better clinical acceptance, A-GAS is developed such that the methodology behind the algorithm of A-GAS resembles the thinking process of a clinician while comparing an abnormal gait with a normal gait. Therefore, the parameters of A-GAS are easy to understand and manipulate.

4) The work includes testing the performance of A-GAS on two joint angle profile configurations. Both the configurations are compatible with the present instruments available in clinics. The first configuration targets gait laboratories with multiple-camera set-up. It includes nine joint angle profiles viz., ankle sagittal, knee sagittal, hip sagittal, pelvic sagittal, hip transverse, pelvic transverse, hip coronal, pelvic coronal and foot transverse. The second configuration targets gait laboratories with Kinect, one camera set-up or goniometers. It includes three joint angle profiles viz., ankle sagittal, knee sagittal and hip sagittal.

The rest of the work is as follows: steps to compute AGAS are described in Section II performance of the proposed assessment system is presented in Section III] a comparison of A-GAS with one rater-based method, the significance of the research, limitations and future plans of A-GAS are discussed in Sections IV finally, the conclusion of the work is drawn in Section $\mathrm{V}$

\section{Methodology}

\section{A. Dataset Description}

This work includes rich datasets for testing the performance of A-GAS on the many possible gait abnormalities. One CP children's and two typically developing (TD) children's, publicly available datasets with a total of 397 subjects are used.

1) $\mathrm{CP}$ children dataset [40]: The dataset was recorded by the clinical motion analysis laboratory of University Hospital Pellenberg with approval of the medical ethics committee of University Hospitals Leuven (ref. s56036) [41], [42]. It consists of gait analysis sessions recorded from 356 children (age 4 to 18 years) with unilateral or bilateral spastic CP (GMFCS level I-III). The proposed work uses only kinematics data from 1719 trials recorded by the Vicon system (Vicon Motion Systems Ltd., Oxford, UK) at self-selected speed. Each trial is a gait cycle with 51 number of samples. A sample plot of the distribution of knee angle's profile is shown in Fig. 2 A.

2) Normal dataset [43], [44]: The dataset was recorded with signed informed consents of the participants and approval of the Institutional Research Ethics Committee of Fondazione Don Carlo Gnocchi. It consists of data from 41 TD children (20 from [43], 21 from [44]) aged 6 to 17 years. This dataset contains a fewer number of subjects when compared to $\mathrm{CP}$ dataset. However, it is found to be correlated with another dataset [45] of $83 \mathrm{TD}$ children with a similar age group [43]. The proposed work uses only kinematics data recorded by a 9-camera motion capture system (SMART system, BTS, Garbagnate Milanese, Italy) at self-selected speed. For further processing, each trial of kinematic data is downsampled from 101 to 51 gait cycle instances to match with CP dataset's sampling frequency. A sample plot of the distribution of knee angle's profile is shown in Fig. 2 A.

Ankle sagittal, knee sagittal, hip sagittal, pelvic sagittal, hip transverse, pelvic transverse, hip coronal, pelvic coronal and foot transverse joint angle profiles are extracted from the datasets (see Fig. 11).

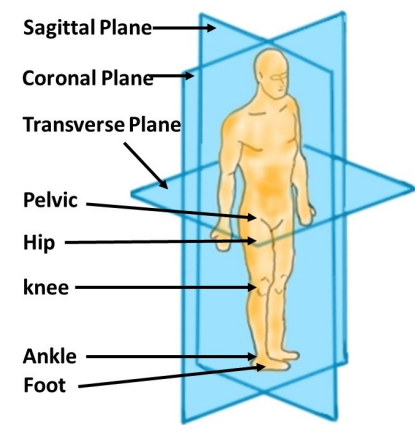

Fig. 1. Graphics illustrating three orthogonal planes with respect to the human body and five segments/joints of the lower portion of human body. The movement of these segments/joints in the three planes during gait generate joint angle profiles.

\section{B. Normal vs abnormal comparison}

For the feasibility of using a probabilistic approach for gait assessment, the assumption that gait angle profiles of CP significantly differ from TD must hold. To verify this assumption, some statistical tests are performed for each joint profile. First, the normality of histograms for $\mathrm{CP}$ gait cycles and TD gait cycles at each cycle instance is verified by Shapiro-Wilk test. Then normal probability density functions (PDFs) are then fitted to the data at each cycle instance with 1719 CP data points and 41 TD data points. A sample plot of PDFs of CP and TD angle profiles at 30th gait cycle instance is shown in Fig. $2 \mathrm{~B}$. Then, the significance of the difference of 
means of CP gait cycles and TD gait cycles at each cycle instance is evaluated by a T-test. $\mathrm{P}$ values obtained by $\mathrm{T}$ test for knee angle profile are shown in Fig. 2D. It shows that the difference in means of $\mathrm{CP}$ gait cycles and TD gait cycles is significant ( $\mathrm{p}$-value less than 0.05) at most of the gait cycle instances for knee joint angle. Similar results are obtained for other joint angle profiles. So we can conclude that the assumption of gait angle profiles of CP significantly differ from TD holds. Therefore, there is a possibility of the existence of a probabilistic solution for quantifying gait abnormality.

\section{Instances weight calculation}

In the previous analysis, at some gait cycle instances, the difference of means of CP gait cycles and TD gait cycles was not significant Fig. 2D. This suggests there is an absence of evidence to reject the null hypothesis statistically. In other words, we do not have the confidence to say that means of $\mathrm{CP}$ gait cycles and TD gait cycles at these cycle instances are different. While at most of the cycle instances, the difference was significant. This suggests, there is sufficient evidence to reject the null hypothesis statistically. In other words, we do have the confidence to say that means of $\mathrm{CP}$ gait cycles and TD gait cycles at these cycle instances are different. Mathematically, the confidence as mentioned above is analogous to the p-value of the T-test. The gait cycle instances with $\mathrm{p}$ values close to one, have lesser significance for distinguishing values of $\mathrm{CP}$ gait cycle with TD gait cycle when compared with the gait cycle instances with p-values close to zero. As it can be observed that significance and p-values are inversely proportional. To quantify this significance, a weight instance vector is defined at all cycle instances. By using a non-linear mapping, the p-value is converted into an instance weight at each gait cycle instance. This non-linear mapping is given by the following equation 1, and its shown in Fig. 2.C.

$$
\text { InstanceWeight }(\text { Pvalue })=1+\frac{(0.25-1)}{1.5^{1 / \text { Pvalue }}}
$$

Instance weight is calculated for all instances and stored in a vector 'I'. The least value of $I$ is set to 0.5 to avoid vanishing the entity's value multiplied with it. The nature of the vector is chosen to be non-linear with a slower descent rate at smaller $\mathrm{p}$-values values to take advantage of the physical significance of p-values.

\section{Computation of A-GAS of a given sample}

An abnormality score A-GAS is computed for representing the degree of abnormality in gait. For a given sample $X$, let us assume that the angle profile of knee joint in sagittal plane at $i t h$ gait cycle instance is denoted by $X_{i}^{k n e e S}$ and the abnormality index of knee angle profile in sagittal plane at $i t h$ gait cycle instance is denoted by $A I I_{i}^{k n e e S}$. So, the expression for computing $A I I_{i}^{k n e e S}$ is given by the following equations 2

$$
A I I_{i}^{k n e e S}=I_{i}^{k n e e S} * L_{i}^{k n e e S}
$$

Where, $I_{i}^{k n e e S}$ is $i t h$ gait cycle instance value of instance weight vector $I^{k n e e S}$ computed for knee joint, $L_{i}^{k n e e S}$ is $i t h$ gait cycle instance value of modified likelihood ratio vector $L^{k n e e S}$ of knee joint and $A I I_{i}^{k n e e S}$ is $i t h$ gait cycle instance value of instance abnormality index vector $A I I^{\text {kneeS }}$ of knee joint. For computation of $L_{i}^{k n e e S}$, likelihood of $X_{i}^{k n e e S}$ for CP gait cycle at $i t h$ instance and TD gait cycle at $i t h$ instance are calculated from their respective fitted PDFs. To elaborate, in step 1, PDFs for CP gait cycles and TD gait cycles are computed for a gait cycle instance. A sample PDF plot is shown in Fig. 3B for gait cycle instace 30 of sample shown in Fig. 3A. In step 2, likelihood of the values of a given sample is noted. In the given example, $X_{30}^{k n e e}=27, \mathrm{\iota}\left(\mu_{30}^{C P}, \sigma_{30}^{C P} \mid 27\right)=$ 0.03367 and $\mathrm{七}\left(\mu_{30}^{T D}, \sigma_{30}^{T D} \mid 27\right)=0.00830$. Here, $\mu_{30}^{C P}$ and $\sigma_{30}^{C P}$ are mean and SD of CP profile value's distribution at the 30th gait cycle instance, respectively. $\mu_{30}^{T D}$ and $\sigma_{30}^{T D}$ are mean and SD of TD profile value's distribution at 30th gait cycle instance, respectively. $€$ is the likelihood function. In step 3, likelihood ratio ( $L R_{i}^{k n e e S}$ ) of TD to $\mathrm{CP}$ is computed by equation 3 . For the sample, likelihood ratio of TD to $\mathrm{CP}$ is $0.008305 / 0.03367=0.2467$.

$$
L R_{i}^{k n e e S}=\frac{£\left(\mu_{i}^{T D}, \sigma_{i}^{T D} \mid X_{i}^{k n e e S}\right)}{\mathrm{Ł}\left(\mu_{i}^{C P}, \sigma_{i}^{C P} \mid X_{i}^{k n e e S}\right)}
$$

In step 4, modified likelihood ratio $L_{i}^{k n e e S}$ is computed by the following equation 4

$$
L_{i}^{k n e e S}=\left\{\begin{array}{ccc}
1-L R_{i}^{k n e e S} & \text { if } & L R_{i}^{k n e e S} \leq 1 \\
0 & \text { if } & L R_{i}^{k n e e S}>1 \\
0 & \text { if } & \mathrm{七}\left(\mu_{i}^{C P}, \sigma_{i}^{C P} \mid X_{i}^{k n e e S}\right)=0
\end{array}\right.
$$

According to the equation 4, the modified likelihood ratio of the given sample is $1-0.2467=0.7533$. Modified likelihood ratio is inspired from Bayes' discrimination rule which states that a sample $x$ is assigned to a group that maximises $\pi_{i} \mathrm{七}_{i}(x)$ is higher, where $\pi_{i}$ is prior probability of group $i$. However, it it not possible to accurately estimate $\pi$ for CP and TD group at each gait cycle instance. Therefore, it is assumed same for both the groups. In the final step, steps 1-4 are repeated for all gait cycle instances to compute $L^{\text {kneeS }}$.

For all nine joint angle profiles, a joint abnormalcy index (i.e. $A I^{\text {joint }}$ ) is calculated by adding $A I I_{i}^{k n e e S}$ at all instances (equation 5).

$$
A I^{k n e e S}=\sum_{i=1}^{51} A I I_{i}^{k n e e S}
$$

These $A I^{\text {joint }}$ are multiplied with their corresponding weight $W^{\text {joint }}$ and summed to compute A-GAS.

$$
A-G A S=\sum_{j=1}^{9} A I^{j} * W^{j}
$$

where, $W^{j}$ is the weight of $j t h$ joint. Weight vector $W$ represents prior probability of abnormality in joints. The values of $\mathrm{W}$ are derived from Table 1 

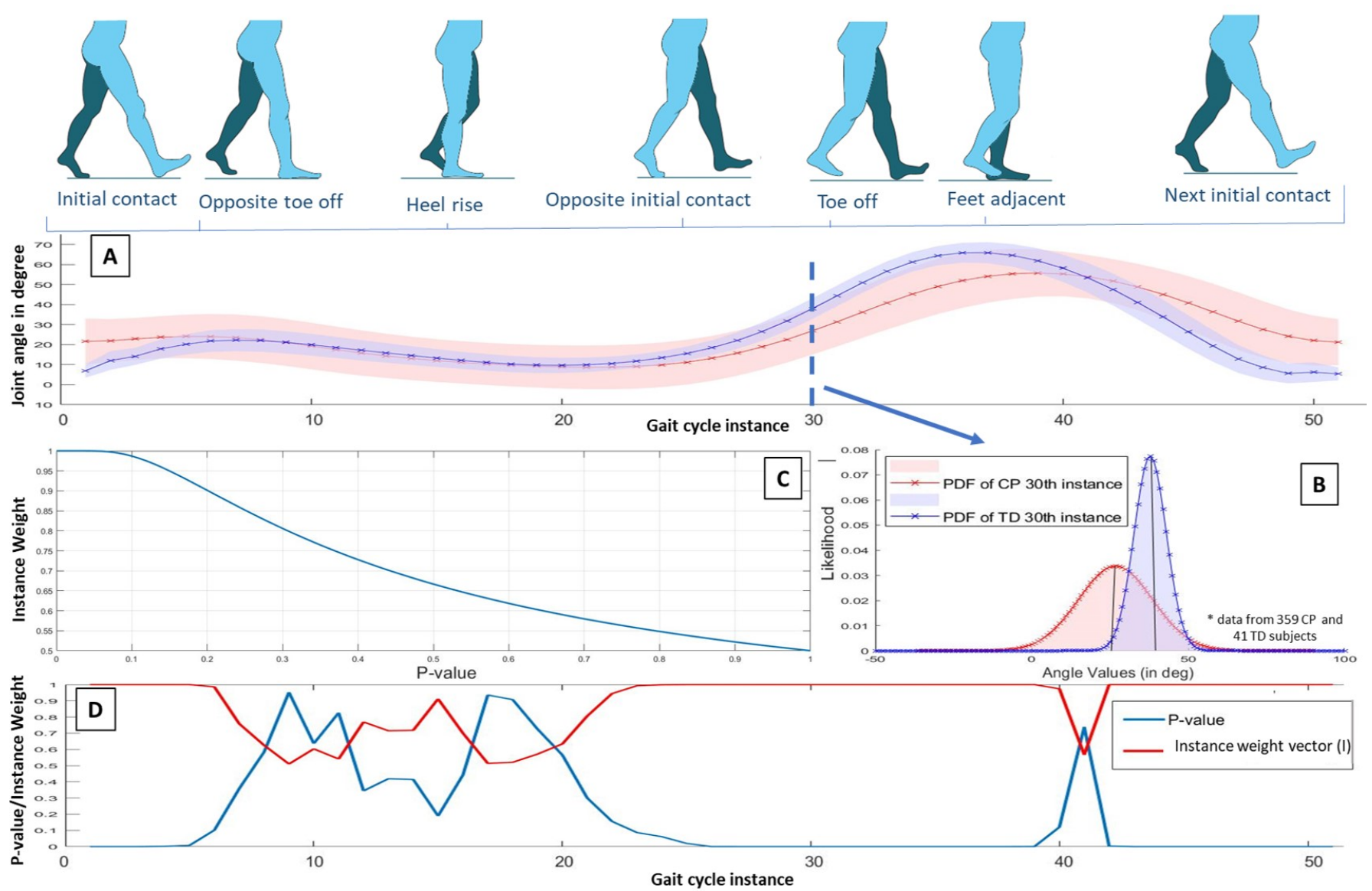

Fig. 2. The top portion of the figure shows different gait events with respect to the right leg (light blue). A: Mean and SD of knee sagittal joint angle profile for CP (red) and TD (blue) subjects is shown. X-axis is gait cycle instance (range 1 to 51) and Y-axis is joint angle in degree. B: PDFs of CP (red) and TD (blue) subjects at 30th gait cycle instance of knee sagittal joint angle profile. C: Plot of the function used to convert P-value from T-test into Instance weight. D: Plot showing P-values (y-axis) and Instance weight vector for each gait cycle instance (x-axis).

TABLE I

NO OR MINOR GAIT DEVIATIONS PERCENT SAMPLE IN CP DATASET FOR VARIOUS JOINT ANGLE PROFILE ACCORDING TO RATINGS OF AN EXPERT [42].

\begin{tabular}{|c|c|}
\hline Joint angle profile & No or minor gait deviations (\%) \\
\hline Knee Sagittal & 23.04 \\
\hline Hip Sagittal & 55.3 \\
\hline Hip Coronal & 62.9 \\
\hline Hip Transverse & 75.4 \\
\hline Ankle Sagittal & 39.16 \\
\hline Pelvic Sagittal & 16.0 \\
\hline Pelvic Coronal & 48.6 \\
\hline Pelvic Transverse & 44.4 \\
\hline Foot Transverse & 66.6 \\
\hline
\end{tabular}

The values from Table 1 are first assigned to W, then linearly maped from 0.5 to 1 to and normalised by dividing $\sum W$. So, the final values of $W$ become $0.941,0.669,0.605,0.5,0.805,1,0.726,0.761$ and 0.574 . A similar reasoning is used for computing A-GAS by using only three joint angle profiles viz., ankle sagittal, knee sagittal and hip sagittal. Thus, the equation for A-GAS is given by the following equation 7

$$
A-G A S=\sum_{j=1}^{3} A I^{j} * W^{j}
$$

The final values of $W$ becomes $1,0.5$ and 0.748 . The pseudocode of calculating A-GAS, AI and AII for a sample $X$ is given in Fig. 4 . For all the analyses, a system having the following specifications is used; Intel(R) Core(TM) i5 6200U CPU @ $2.30 \mathrm{GHz}, 4$ GB DDR4 RAM, NVIDIA GeForce 930MX graphic engine, 64-bit Windows 10 Operating System and Matlab 2020a platform.

\section{RESULTS}

\section{A. A sample report of A-GAS}

By following the steps mentioned in the Section II report of A-GAS is generated for a sample CP subject (see Fig. 5).

The report of A-GAS has four key features. First, it provides an easily interpretable comprehensive gait assessment. Second, it utilises the unique properties of each gait cycle instance to compute the corresponding AII. Third, it provides abnormality indices at three levels for a better understanding of gait pathology. Fourth, it uses knowledge of both $\mathrm{CP}$ and TD distributions to compute $A I I$.

\section{B. Comparison of A-GAS for $C P$ and TD subjects}

To understand the performance of A-GAS an analysis is performed. A-GAS is computed for all CP and TD subjects. 


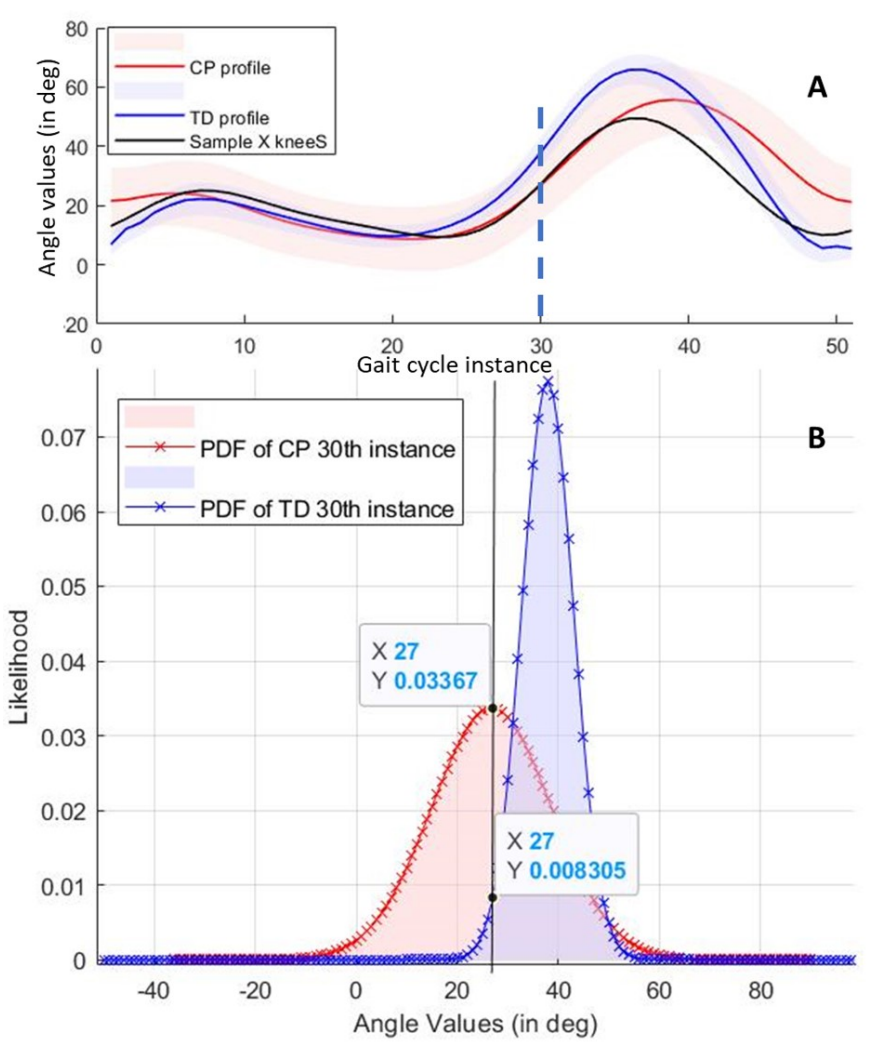

Fig. 3. A: Knee sagittal joint angle profile of a sample $X^{\text {kneeS }}$ (black), mean and SD of CP (red) subjects and mean and SD (blue) of TD subjects is shown. B: Position of $X_{30}^{k n e e S}$ in PDFs of CP (red) and TD (blue) subjects at 30th gait cycle instance of knee sagittal joint angle profile.

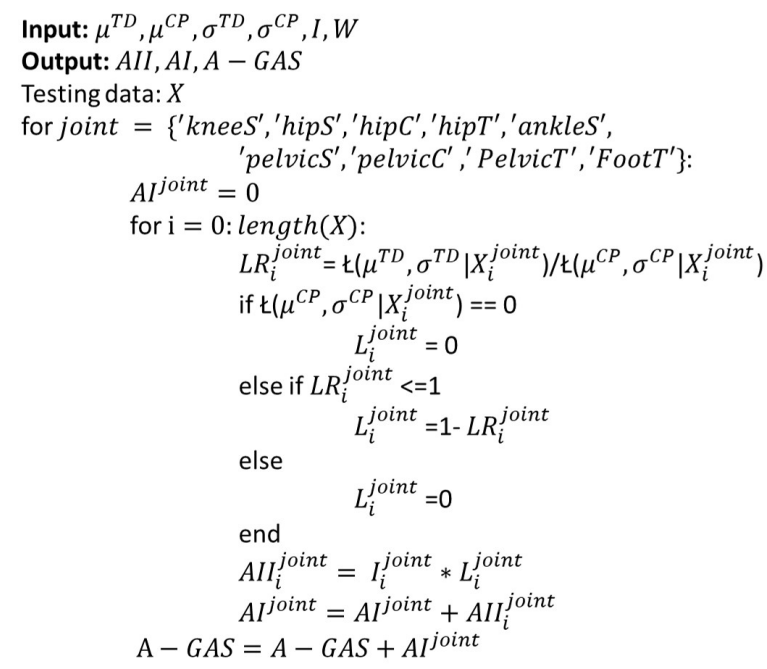

Fig. 4. The pseudo-code of calculating A-GAS, AI and AII for a sample $X$.

Fig. 6 shows PDFs of CP and TD subjects. It can be interpreted from the Fig. 6 that A-GAS value of TD subjects is less than most of the CP subjects. The overlapping areas of PDFs is negligible, and by setting a decision criterion at around 0.13 , A-GAS can accurately classify subjects into TD and CP.

A similar analysis is performed for individual joints. Fig. 7 shows PDFs of CP and TD subjects for all nine joints. It can be interpreted from the Fig. 7 that $A I$ value of TD subjects is less than most of the $\mathrm{CP}$ subjects at all joints. However, there are noticeable overlapping of PDFs of $A I$ for $\mathrm{CP}$ and TD subjects at hip coronal, pelvic coronal and pelvic transverse joint angle profiles. There can be multiple possible reasons for these overlapping. One reason can be no or less deviation of $\mathrm{CP}$ gait angle profile from TD gait angle profile in many $\mathrm{CP}$ subjects at hip coronal, pelvic coronal and pelvic transverse joint angle profiles. This reasoning is also supported by results of [42], where $62.9 \% \mathrm{CP}$ hip coronal angle profile, $48.6 \%$ pelvic coronal angle profile and $44.4 \%$ pelvic traverse angle profile showed no minor gait deviations. Another possible reason can be the induction of minor abnormality in TD subjects at hip coronal, pelvic coronal and pelvic transverse joint angle profiles due to measurement errors. The range of motion of these joints is very small $\left(5^{\circ}-20^{\circ}\right)$, and small error in measurement can leads to a large deviation from normal gait angle profile.

\section{Comparison of assessments}

The establishment of an instrumented gait laboratory needs plenty of money and space. Moreover, recording and processing of 3-dimensional signal takes time [46]. However, low-cost equipments such as Kinect, one-camera set-up and goniometer can be easily used for both small clinics and personal use. Therefore, an experiment is performed to propose a combination of three joint angle profiles that can replace current nine joint angle profile configuration effectively. A configuration having knee sagittal, hip sagittal and ankle sagittal joint angle profile is used for developing a three joint angle profile based score because its performance is found to be better than all other possible combinations. At all samples, BlandAltman plot [47] is calculated for three joint angle profiles configuration (say A-GAS 1) and nine joint angle profiles configuration (say A-GAS 2). Fig. 8 shows, around 96\% difference (A-GAS - 1 A-GAS 2) lies in 95\% confidence level range. This suggests that both the configurations can be used interchangeably for clinical use [47].

\section{Discussion}

\section{A. Comparison of A-GAS with present rating based scale}

As discussed in the Section [1] most of the present scales are based on raters. Since all rating-based scale has its pros and cons, it is very difficult to select the best scale among them. To address this problem, a consensus study was performed by Angela Nieuwenhuys et al. [48]. Thirteen experts from eight gait laboratories identified 49 clinical assessment relevant joint motion patterns in children with CP. Each trial of the $\mathrm{CP}$ dataset used in the study is rated by one of the two expert rater. '0'rating for a joint motion pattern denotes no or minor deviation from TD, while, rating other than ' 0 'denotes a specific gait abnormality. This rater-based method provides discrete value of rating while A-GAS provides a continuous score. Therefore, for comparison, all trials are divided into two classes; one with '0'rating and other with non- '0'ratings. Fig. 9 shows histograms of A-GAS for both the classes at each joint angle profile. In all the joint angle profiles, mean of AGAS for class with '0'rating is less than mean of A-GAS for 

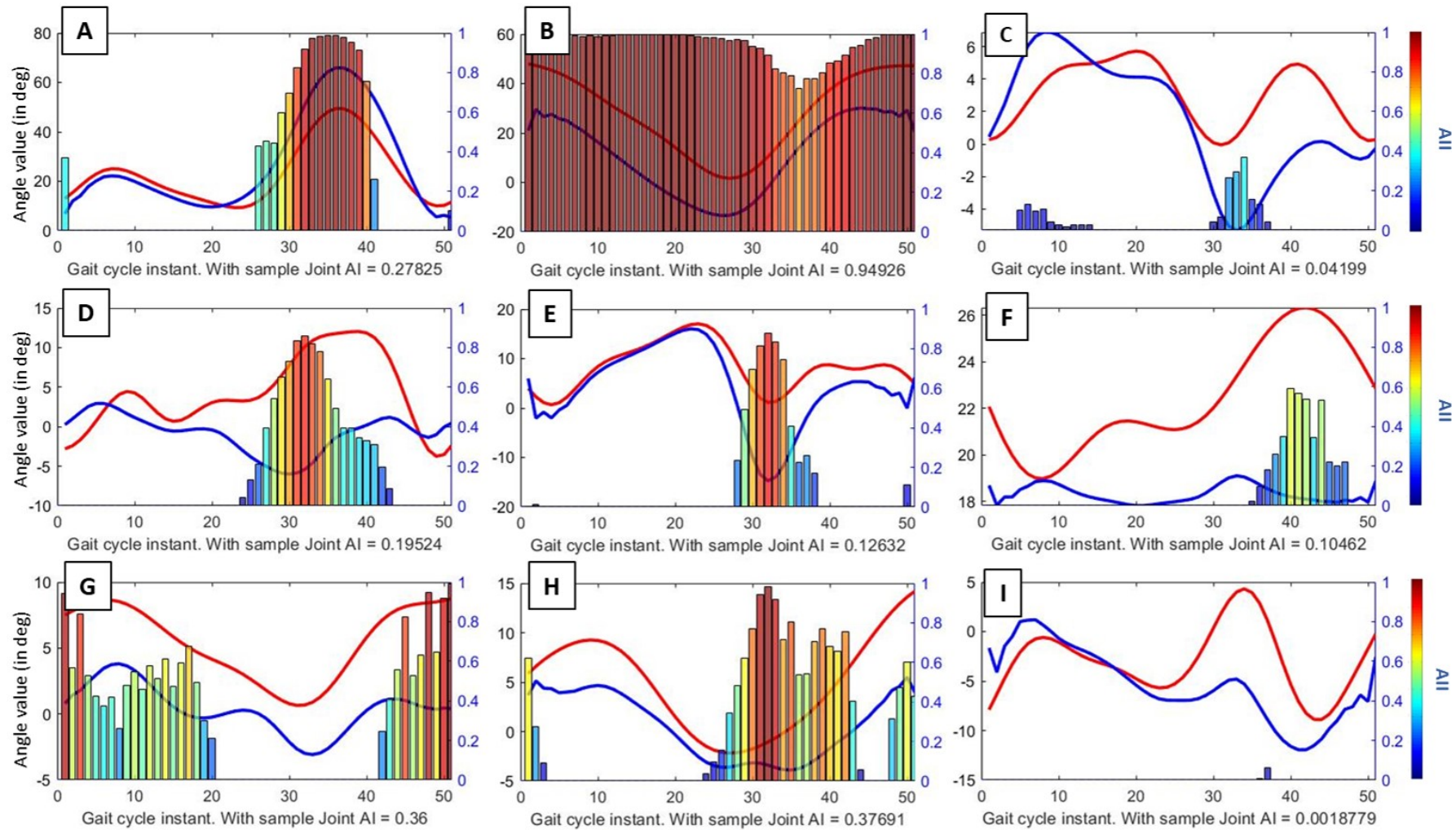

Gait cycle instant. With sample Joint $\mathrm{Al}=0.10462$

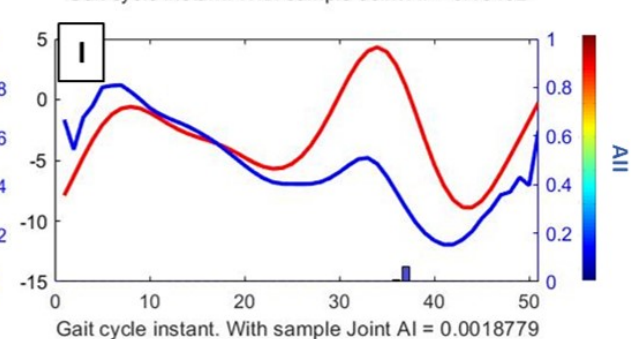

A-GAS $=0.4791$

Fig. 5. Report of A-GAS consists of nine subplots. Each subplot corresponds to one joint angle profile; A: knee sagittal, B: hip sagittal, C: hip coronal, D: hip transverse, E: ankle sagittal, F: pelvic sagittal, G: pelvic coronal, H: pelvic transverse and I: foot transverse. In a subplot, blue curve represents mean normal joint profile, red curve represents sample joint angle profile, and height and colour of bar plot represent the magnitude of instance abnormality index (AII) at an instance, y-left axis represents angle value (in degree), y-right represents AII, x-axis represents gait cycle instance and numerical value at the bottom of each subplot represents joint AI. A-GAS of the sample is written at the bottom of the plot.

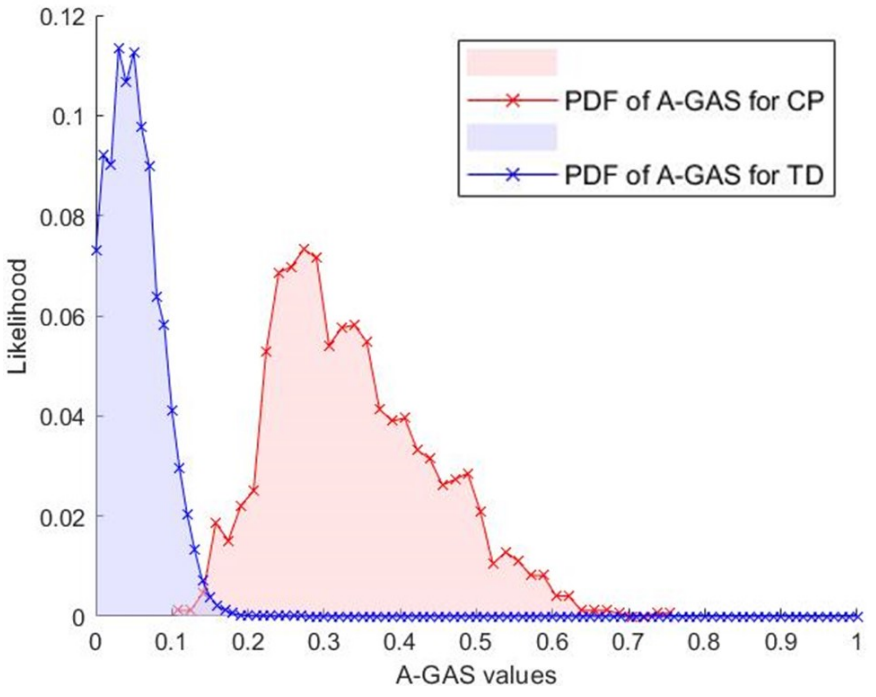

Fig. 6. PDFs of A-GAS calculated for CP and TD samples.

class with non- '0'rating. In a scenario when both assessments are perfect we expect zero overlapping between the histograms with zero A-GAS value for all trials of class with '0'rating. However, in the Fig. 9 we can observe a lot of overlapping between the histograms at some joint angle profiles. The possible reason for overlapping can be, incompleteness and in- sensitiveness of the rating based scale. Since, the rating based scale exploited a limited number of gait abnormal patterns to quantify abnormalities, sometimes it fails to capture abnormal patterns. This limitation of rater-based method contribute to its incompleteness to understand all possible types of gait abnormalities. Non-zero value of A-GAS value for trials of class with '0'rating supports this argument. Furthermore, the rater-based method is in-sensitive to minor changes in gait angle profile because it is based on a discrete scale. For example, the same rating will be provided to a knee joint in sagittal with minor knee hyper-extension and a knee joint in sagittal with major knee hyper-extension [42]. While A-GAS is sensitive to even minor pathological changes as it provides a continuous reading of gait abnormalities.

As mentioned in the literature review sections, previously many researchers attempted to quantify gait anomalies. However, a direct comparison of A-GAS with these methods is not feasible due to different datasets and following four reasons. First, these methods either provide a global abnormality index or perform classification into a small number of gait abnormalities, while A-GAS provides joint-specific gait assessment score. Second, these methods used either used IMUs or 3-D cloud data for recording gait, while A-GAS uses joint angle profiles of the joints. Third, the applicability of these methods is not tested in clinical settings, therefore, their credibility is questionable. Fourth, a completely different experimental 

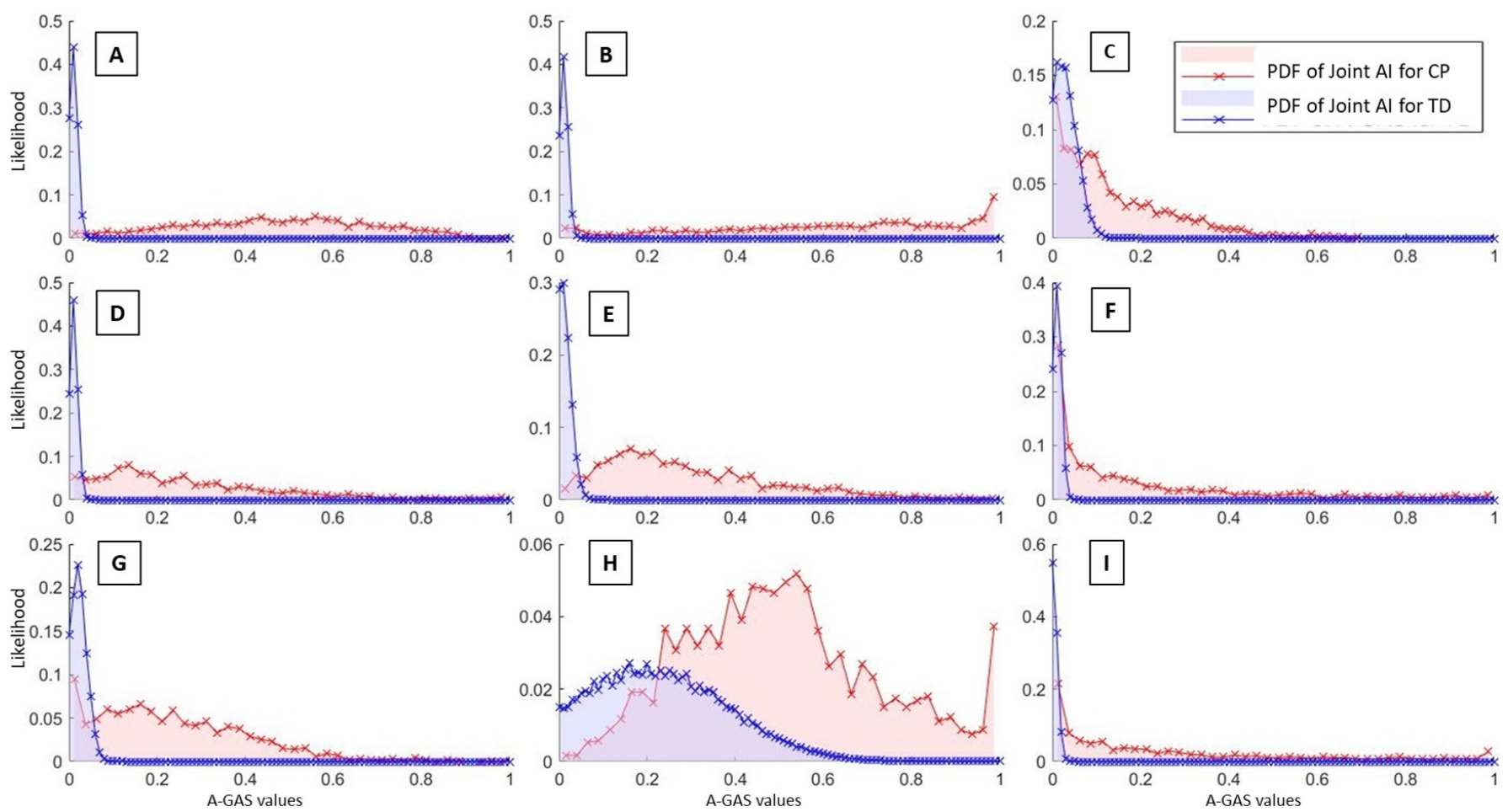

Fig. 7. The figure consists of nine subplots. Each subplot corresponds to one joint angle profile; A: knee sagittal, B: hip sagittal, C: hip coronal, D: hip transverse, E: ankle sagittal, F: pelvic sagittal, G: pelvic coronal, H: pelvic transverse and I: foot transverse. In a subplot, blue curve represents PDF of joint abnormality index (AI) of TD sample for its corresponding joint angle profile and red curve represents PDF of AI of CP sample for its corresponding joint angle profile.

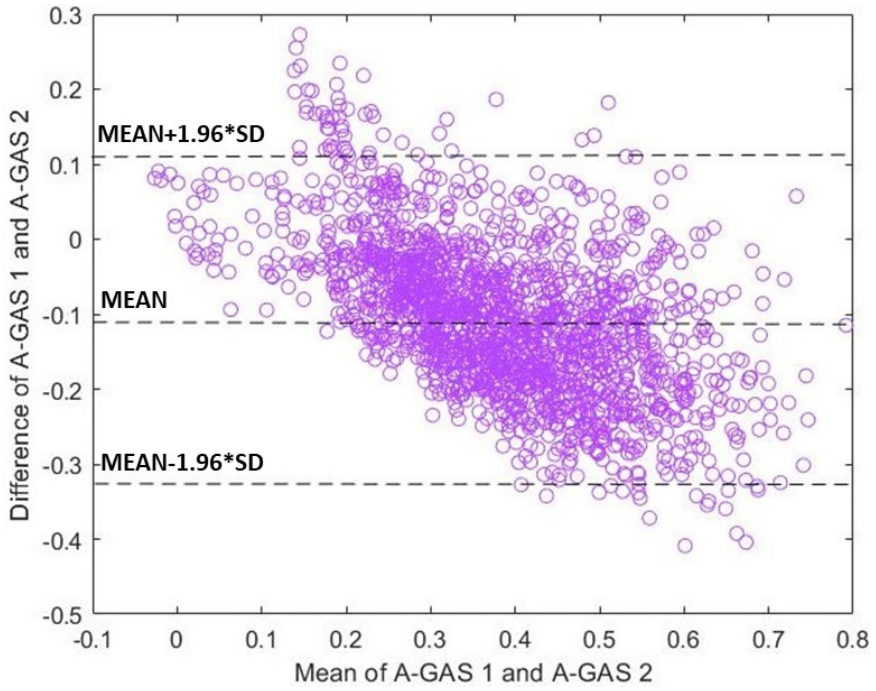

Fig. 8. Bland- Altman plot for three joint angle profiles configuration (say A-GAS 1) and nine joint angle profiles configuration (say A-GAS 2). (A-GAS - 1 A-GAS 2) difference for 1690 samples out of 1760 lies in $95 \%$ confidence level range.

protocol and instrumentation is needed to implement each of these methods.

\section{B. Significance of the research, limitations and future plans}

Gait management in CP and other pathological gait disorders is a long process. It is crucial to track the progress of the

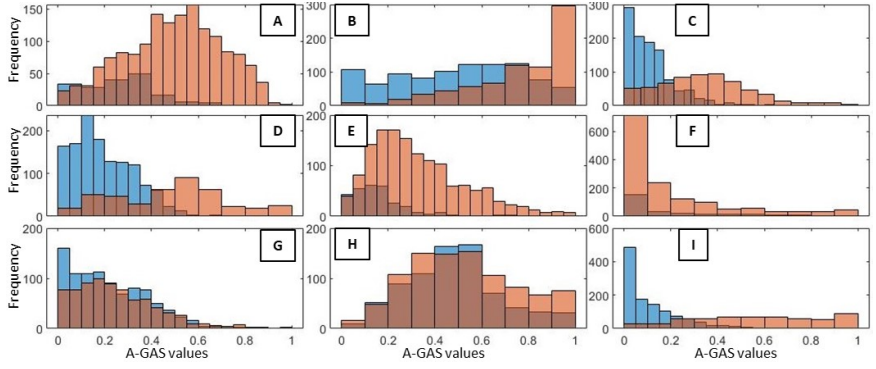

Fig. 9. The figure consists of nine subplots. Each subplot corresponds to one joint angle profile; A: knee sagittal, B: hip sagittal, C: hip coronal, D: hip transverse, E: ankle sagittal, F: pelvic sagittal, G: pelvic coronal, H: pelvic transverse and I: foot transverse. In a subplot, the blue region represents the histogram of joint abnormality index (AI) of trials of class with '0'rating for its corresponding joint angle profile and red curve represents PDF of AI of trials of class with non-'0'rating for its corresponding joint angle profile.

process by timely recording patient's gait activities. However, rating a limited number of gait events with discrete values may lead to poor sensitivity of the method [27]. Moreover, during this long process of treatment, a patient may have to change their doctor. However, as most of the ways are subjective, the therapy may suffer after switching doctors. In addition, the subjectivity of these methods may lead to poor reliability, specificity, sensitivity, and validity [49]. Despite many possible solutions for developing a robust automated gait assessment using a deep learning-based method, we choose a conventional approach due to three reasons. First, it is very difficult to interpret/visualise the computations by using a deep 
neural network to predict gait assessment score. Conversely, a probabilistic approach based on simple computation provides sense to the intermediate computations. Such transparency in computations increases the reliability and clinical acceptance of the method. Second, an optimum solution of a deep learning-based approaches corresponds to a local minima of the loss function. Use of the same network architecture on a similar problem do not guarantee the convergence of the solution. In other words, deep learning-based approaches are problem and training data specific. However, simple tools of statistics and probability are problem and data invariant. AGAS is a genialised approach that can also be used for other modalities of gait data, such as gait kinetics. Third, implementation and understanding of deep learning-based approaches require high-performance computers (including GPUs/TPUs) and expertise in a specific field. While computations of AGAS can be done on simple computers/smartphones. Also, elementary knowledge of mathematics and programming is sufficient for its implementation and understanding.

The work proposed an automated method for gait assessment with a great potential for clinical use. However, there are a few limitations of this work. A-GAS's performance has only been tested on $\mathrm{CP}$ a dataset; its performance on other gait pathologies needs to be tested. Also, the feasibility of A-GAS need to be tested in clinical environments. Since the proposed method extracts decision parameters from the normal and abnormal data, its results depend on the population of the datasets. Moreover, as it compares gait cycle instances of a given sample with normal and abnormal datasets, the sampling frequency of the datasets should be matched with the sample. Furthermore, A-GAS can quantify abnormality in a lower limb, so it does not directly account for gait asymmetry. However, by comparing A-GAS, AI, and AII of both the legs, an index for abnormality can be derived. In future work, we will test A-GAS in a clinical setting; by understanding doctors' needs, some additional features will also be added. A collaborated artificial intelligence would be developed in future to suggest possible causes of the gait abnormality and interventions needed for its treatment. In addition, A-GAS will be tested for tracking the progress of rehabilitation in various gait disorders.

\section{CONCLUSiON}

In conclusion, the work tries to improve the method of diagnosing gait abnormalities in $\mathrm{CP}$ by presenting an automated, comprehensive gait assessment score for objectively quantifying gait abnormalities in a lower limb. Performance of A-GAS is verified on kinematic data of $356 \mathrm{CP}$ and 41 TD children, which suggests great potential of its use in clinical settings. A graphical representation of the gait assessment report, including AII, AI, and A-GAS is generated to understand the results in a more interactive way. Moreover, the study compares the performance of A-GAS with a present rating-based gait assessment scores on the same dataset to understand fundamental differences between them. Lastly, the degree of agreement between values of A-GAS's for highcost multi-camera set-up using nine joint angle profiles and values of A-GAS for low-cost single camera set-up using three joint angle profiles is computed. Results suggest both the setups can be used interchangeably, as there are no significant differences in performance of A-GAS for both the set-ups.

\section{REFERENCES}

[1] W. Pirker and R. Katzenschlager, "Gait disorders in adults and the elderly," Wiener Klinische Wochenschrift, vol. 129, no. 3, pp. 81-95, 2017.

[2] M. L. Aisen, D. Kerkovich, J. Mast, S. Mulroy, T. A. Wren, R. M. Kay, and S. A. Rethlefsen, "Cerebral palsy: clinical care and neurological rehabilitation,” The Lancet Neurology, vol. 10, no. 9, pp. 844-852, 2011.

[3] S. Armand, G. Decoulon, and A. Bonnefoy-Mazure, "Gait analysis in children with cerebral palsy," EFORT open reviews, vol. 1, no. 12, pp. 448-460, 2016.

[4] A. A. Kareem, "Use of botulinum toxin a in cerebral palsy," Cerebral Palsy-Clinical and Therapeutic Aspects, pp. 95-112, 2018.

[5] C. Rathinam, A. Bateman, J. Peirson, and J. Skinner, "Observational gait assessment tools in paediatrics-a systematic review," Gait \& posture, vol. 40, no. 2, pp. 279-285, 2014.

[6] F. Moissenet and S. Armand, "Qualitative and quantitative methods of assessing gait disorders," Orthopedic management of children with cerebral palsy: a comprehensive approach, pp. 215-239, 2015.

[7] D. H. Sutherland and J. R. Davids, "Common gait abnormalities of the knee in cerebral palsy." Clinical orthopaedics and related research, no. 288, pp. 139-147, 1993.

[8] T. Winters, J. Gage, and R. Hicks, "Gait patterns in spastic hemiplegia in children and young adults," J Bone Joint Surg Am, vol. 69, no. 3, pp. 437-441, 1987.

[9] J. Rodda and H. Graham, "Classification of gait patterns in spastic hemiplegia and spastic diplegia: a basis for a management algorithm," European journal of neurology, vol. 8, pp. 98-108, 2001.

[10] T. F. Novacheck, J. L. Stout, and R. Tervo, "Reliability and validity of the gillette functional assessment questionnaire as an outcome measure in children with walking disabilities,' Journal of pediatric orthopaedics, vol. 20, no. 1, p. 75, 2000.

[11] H. K. Graham, A. Harvey, J. Rodda, G. R. Nattrass, and M. Pirpiris, "The functional mobility scale (fms)," Journal of Pediatric Orthopaedics, vol. 24, no. 5, pp. 514-520, 2004.

[12] D. J. Russell, P. L. Rosenbaum, D. T. Cadman, C. Gowland, S. Hardy, and S. Jarvis, "The gross motor function measure: a means to evaluate the effects of physical therapy," Developmental Medicine \& Child Neurology, vol. 31, no. 3, pp. 341-352, 1989.

[13] J. W. Varni, T. M. Burwinkle, S. J. Berrin, S. A. Sherman, K. Artavia, V. L. Malcarne, and H. G. Chambers, "The pedsql in pediatric cerebral palsy: reliability, validity, and sensitivity of the generic core scales and cerebral palsy module," Developmental medicine and child neurology, vol. 48, no. 6, pp. 442-449, 2006.

[14] S. M. Haley, W. J. Coster, Y.-C. Kao, H. M. Dumas, M. A. FragalaPinkham, J. M. Kramer, L. H. Ludlow, and R. Moed, "Lessons from use of the pediatric evaluation of disability inventory (pedi): Where do we go from here?" Pediatric physical therapy: the official publication of the Section on Pediatrics of the American Physical Therapy Association, vol. 22, no. 1, p. 69, 2010.

[15] P. Thomason, A. Tan, A. Donnan, J. Rodda, H. K. Graham, and U. Narayanan, "The gait outcomes assessment list (goal): validation of a new assessment of gait function for children with cerebral palsy," Developmental Medicine \& Child Neurology, vol. 60, no. 6, pp. 618623, 2018.

[16] N. L. Young, J. I. Williams, K. K. Yoshida, and J. G. Wright, "Measurement properties of the activities scale for kids," Journal of clinical epidemiology, vol. 53, no. 2, pp. 125-137, 2000.

[17] H. S. Read, M. E. Hazlewood, S. J. Hillman, R. J. Prescott, and J. E. Robb, "Edinburgh visual gait score for use in cerebral palsy," Journal of pediatric orthopaedics, vol. 23, no. 3, pp. 296-301, 2003.

[18] B. Toro, C. J. Nester, and P. C. Farren, "Inter-and intraobserver repeatability of the salford gait tool: an observation-based clinical gait assessment tool," Archives of physical medicine and rehabilitation, vol. 88, no. 3, pp. 328-332, 2007.

[19] T. F. Novacheck, J. P. Trost, and S. Sohrweide, "Examination of the child with cerebral palsy," Orthopedic Clinics of North America, vol. 41, no. 4, pp. 469-488, 2010, orthopedic Management of Cerebral Palsy. [Online]. Available: https://www.sciencedirect.com/ science/article/pii/S0030589810000647 
[20] K. G. Maathuis, C. P. van der Schans, A. Van Iperen, H. S. Rietman, and J. H. Geertzen, "Gait in children with cerebral palsy: observer reliability of physician rating scale and edinburgh visual gait analysis interval testing scale," Journal of Pediatric Orthopaedics, vol. 25, no. 3, pp. 268-272, 2005.

[21] P. Araújo, R. Kirkwood, and E. Figueiredo, "Validity and intra-and interrater reliability of the observational gait scale for children with spastic cerebral palsy," Brazilian Journal of Physical Therapy, vol. 13, pp. $267-$ 273, 2009.

[22] T. A. Wren, S. A. Rethlefsen, B. S. Healy, K. P. Do, S. W. Dennis, and R. M. Kay, "Reliability and validity of visual assessments of gait using a modified physician rating scale for crouch and foot contact," Journal of Pediatric Orthopaedics, vol. 25, no. 5, pp. 646-650, 2005.

[23] B. Toro, C. J. Nester, and P. C. Farren, "The development and validity of the salford gait tool: an observation-based clinical gait assessment tool," Archives of physical medicine and rehabilitation, vol. 88, no. 3, pp. 321-327, 2007.

[24] E. Dugan, F. Trachtenberg, and M. A. Hall, "Development of abbreviated measures to assess patient trust in a physician, a health insurer, and the medical profession," BMC health services research, vol. 5, no. 1, pp. $1-7,2005$.

[25] W. E. Dickens and M. F. Smith, "Validation of a visual gait assessment scale for children with hemiplegic cerebral palsy," Gait \& posture, vol. 23, no. 1, pp. 78-82, 2006.

[26] A. H. Mackey, G. L. Lobb, S. E. Walt, and N. S. Stott, "Reliability and validity of the observational gait scale in children with spastic diplegia," Developmental medicine and child neurology, vol. 45, no. 1, pp. 4-11, 2003.

[27] M. Galli, V. Cimolin, C. Rigoldi, and G. Albertini, "Quantitative evaluation of the effects of ankle foot orthosis on gait in children with cerebral palsy using the gait profile score and gait variable scores," Journal of Developmental and Physical Disabilities, vol. 28, no. 3, pp. 367-379, 2016.

[28] K. Hori, Y. Mao, Y. Ono, H. Ora, Y. Hirobe, H. Sawada, A. Inaba, S. Orimo, and Y. Miyake, "Inertial measurement unit-based estimation of foot trajectory for clinical gait analysis," Frontiers in physiology, vol. 10 , p. $1530,2020$.

[29] J. Gao, P. Gu, Q. Ren, J. Zhang, and X. Song, "Abnormal gait recognition algorithm based on lstm-cnn fusion network," IEEE Access, vol. 7, pp. $163180-163190,2019$.

[30] T.-N. Nguyen and J. Meunier, "Applying adversarial auto-encoder for estimating human walking gait abnormality index," Pattern Analysis and Applications, vol. 22, no. 4, pp. 1597-1608, 2019.

[31] T.-N. Nguyen, H.-H. Huynh, and J. Meunier, "Estimating skeleton-based gait abnormality index by sparse deep auto-encoder," in 2018 IEEE Seventh International Conference on Communications and Electronics (ICCE). IEEE, 2018, pp. 311-315.

[32] X. Chen, S. Liao, S. Cao, D. Wu, and X. Zhang, "An accelerationbased gait assessment method for children with cerebral palsy," Sensors, vol. 17, no. 5, p. 1002, 2017.

[33] C. Kirtley, Clinical gait analysis: theory and practice. Elsevier Health Sciences, 2006.

[34] R. Baker, "Gait analysis methods in rehabilitation," Journal of neuroengineering and rehabilitation, vol. 3, no. 1, pp. 1-10, 2006.

[35] A. Assi, I. Ghanem, F. Lavaste, and W. Skalli, "Gait analysis in children and uncertainty assessment for davis protocol and gillette gait index," Gait \& Posture, vol. 30, no. 1, pp. 22-26, 2009.

[36] A. Muro-De-La-Herran, B. Garcia-Zapirain, and A. Mendez-Zorrilla "Gait analysis methods: An overview of wearable and non-wearable systems, highlighting clinical applications," Sensors, vol. 14, no. 2, pp. 3362-3394, 2014

[37] K. J. Noonan, S. Halliday, R. Browne, S. O'Brien, K. Kayes, and J. Feinberg, "Interobserver variability of gait analysis in patients with cerebral palsy," Journal of Pediatric Orthopaedics, vol. 23, no. 3, pp. 279-287, 2003.

[38] G. Gorton, D. Hebert, and B. Goode, "Assesment of the kinematic variability between 12 shriners motion analysis laboratories," in 6th Annual Meeting of the Gait and Clinical Movement Analysis Society, Sacramento, California, Gait and Posture, 2001.

[39] U. G. Narayanan, "The role of gait analysis in the orthopaedic management of ambulatory cerebral palsy," Current opinion in pediatrics, vol. 19, no. 1, pp. 38-43, 2007.

[40] T. D. Laet, A. Nieuwenhuys, E. Papageorgiou, and K. Desloovere, "3D gait analysis data of children with $\mathrm{CP}$ used for gait classification," 42017. [Online]. Available: https://figshare.com/articles/dataset/3D_gait_analysis_data_ of_children_with_CP_used_for_gait_classification/4877432
[41] A. Nieuwenhuys, E. Papageorgiou, G. Molenaers, D. Monari, T. De Laet, and K. Desloovere, "Inter-and intrarater clinician agreement on joint motion patterns during gait in children with cerebral palsy," Developmental Medicine \& Child Neurology, vol. 59, no. 7, pp. 750-755, 2017.

[42] A. Nieuwenhuys, E. Papageorgiou, K. Desloovere, G. Molenaers, and T. De Laet, "Statistical parametric mapping to identify differences between consensus-based joint patterns during gait in children with cerebral palsy," PLoS One, vol. 12, no. 1, p. e0169834, 2017.

[43] G. Bovi, M. Rabuffetti, P. Mazzoleni, and M. Ferrarin, "A multiple-task gait analysis approach: kinematic, kinetic and emg reference data for healthy young and adult subjects," Gait \& posture, vol. 33, no. 1, pp. 6-13, 2011.

[44] T. Lencioni, I. Carpinella, M. Rabuffetti, A. Marzegan, and M. Ferrarin, "Human kinematic, kinetic and emg data during different walking and stair ascending and descending tasks," Scientific data, vol. 6, no. 1, pp. $1-10,2019$.

[45] M. H. Schwartz, A. Rozumalski, and J. P. Trost, "The effect of walking speed on the gait of typically developing children," Journal of biomechanics, vol. 41, no. 8, pp. 1639-1650, 2008.

[46] R. Bajpai and D. Joshi, "Movenet: A deep neural network for joint profile prediction across variable walking speeds and slopes," IEEE Transactions on Instrumentation and Measurement, vol. 70, pp. 1-11, 2021.

[47] C. Bunce, "Correlation, agreement, and bland-altman analysis: statistical analysis of method comparison studies," American journal of ophthalmology, vol. 148, no. 1, pp. 4-6, 2009

[48] A. Nieuwenhuys, S. Õunpuu, A. Van Campenhout, T. Theologis, J. De Cat, J. Stout, G. Molenaers, T. De Laet, and K. Desloovere, "Identification of joint patterns during gait in children with cerebral palsy: a delphi consensus study," Developmental Medicine \& Child Neurology, vol. 58, no. 3, pp. 306-313, 2016.

[49] B. Toro, C. Nester, and P. Farren, "A review of observational gait assessment in clinical practice," Physiotherapy theory and practice, vol. 19 , no. 3, pp. 137-149, 2003.

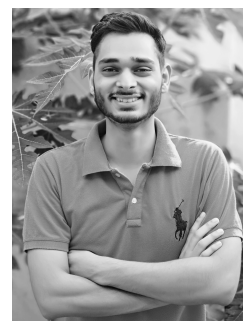

Rishabh Bajpai is currently a $\mathrm{PhD}$ scholar at the Centre for Biomedical Engineering, Indian Institute of Technology (IIT) Delhi, and All India Institute of Medical Sciences (AIIMS) Delhi, India. He received his B.Tech degree in Mechanical Engineering from PDPM Indian Institute of Information Technology, Design and Manufacturing Jabalpur, Madhya Pradesh, India. He has been working in the area of Human-Computer Interface, Rehabilitation engineering, Neuroscience, Electroencephalography and development of biomedical instrumentation for applications specific to assistive devices for disabled.

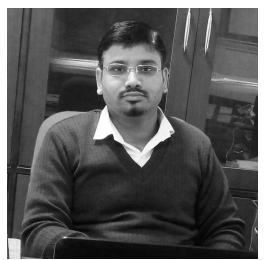

Deepak Joshi is a faculty at Biomedical Engineering at IIT Delhi and All India Institute of Medical Sciences Delhi. He received his PhD in biomedical engineering from Indian Institute of Technology (IIT) Delhi. He has been working in the area of neuroprosthetics and neurorehabilitation for last nearly fourteen years. Before joining to IIT Delhi, he worked at various places including National University of Singapore, Newcastle University UK, and University of Oregon, USA. Dr. Joshi's current research work combines experimental and computational techniques to understand the neural correlates during balancing and seamless transitions during walking. Besides that, he is actively engaged in projects related to development of wearable devices for applications specific to diagnosis of neuromuscular disorders, assistive devices for elderly and disabled, and biofeedback for rehabilitation in stroke patients. 